\title{
GENERIC PROPERTIES OF HOMOGENEOUS RICCI SOLITONS
}

\author{
LUCA FABRIZIO DI CERBO
}

\begin{abstract}
We discuss the geometry of homogeneous Ricci solitons. After showing the non-existence of compact homogeneous and non-compact steady homogeneous solitons, we concentrate on the study of left invariant Ricci solitons. We show that, in the unimodular case, the Ricci soliton equation does not admit solutions in the set of left invariant vector fields. We prove that a left invariant soliton of gradient type must be a Riemannian product with nontrivial Euclidean de Rham factor. As an application of our results we prove that any generalized metric Heisenberg Lie group is a non-gradient left invariant Ricci soliton of expanding type.
\end{abstract}

\section{INTRODUCTION}

A Ricci soliton is a solution of the Ricci flow

$$
\begin{aligned}
& \frac{\partial g}{\partial t}=-2 R i c_{g} \\
& g(0)=g_{0}
\end{aligned}
$$

that changes only by diffeomorphisms and scale. More precisely, $g(t)$ is called Ricci soliton if there exist a smooth function $\sigma(t)$ and a 1-parameter family of diffeomorphisms $\left\{\psi_{t}\right\}$ of $M^{n}$ such that $g(t)=\sigma(t) \psi_{t}^{*}\left(g_{0}\right)$ with $\sigma(0)=1$ and $\psi_{0}=$ $i d_{M^{n}}$. It is easy to see that this condition is equivalent to requiring that the initial metric $g_{0}$ satisfies the following Einstein like identity

$$
-2 R i c_{g_{0}}=2 \lambda g_{0}+L_{X} g_{0}
$$

where $\lambda$ is a real number and $X$ a complete vector field; for the details we refer to [5. We then say that the soliton is expanding, shrinking, or steady if $\lambda>0, \lambda<0$, or $\lambda=0$ respectively. Finally, if the vector field $X$ is the gradient field of a smooth function $f$, one says that the soliton is a gradient Ricci soliton.

The soliton theories in the compact and complete non-compact cases are drastically different. In the compact case it is easy to prove the non-existence of steady and expanding solitons, see [5]; while a result of Perelman [28] ensures that any shrinking soliton must be of gradient type. Moreover, there are no solitons in dimension two and three as proved by Hamilton and Ivey [11, [12. Finally, it is interesting to notice that nontrivial examples of compact gradient Ricci solitons were actually constructed by Koiso in [18. These examples start in dimension four and are Kähler. For more details about compact Kähler-Ricci solitons we refer to [5].

The complete non-compact theory is much richer. Besides the shrinking and expanding Gaussian solitons on $\mathbb{R}^{n}$ and the cylinder soliton on $S^{n} \times \mathbb{R}(n \geq 2)$ it is worthwhile to mention the Hamilton "cigar" soliton on $\mathbb{R}^{2}$, the radially symmetric steady Bryant solitons on $\mathbb{R}^{n}(n \geq 3)$ and their generalization by Ivey, who constructed Ricci solitons on doubly warped products, see [5, 4, [13. It turns out that all these examples are of gradient type.

*Supported in part by the Simons Foundation. 
A completely different family of solitons has been discovered by Lauret, see 19] and the more update 21]. In [19, Lauret searches for a notion weakening the Einstein condition for a left invariant metric $g$ on a nilpotent Lie group $N^{n}$. This is motivated by the fact that nilpotent Lie groups do not admit any left invariant Einstein metric, see 24]. The Ricci soliton condition (2) clearly provides a substitute candidate and Lauret proves that, given a nilpotent Lie group $N^{n}$ with Lie algebra $\mathfrak{n}$ and a left invariant metric $g$, then (2) is satisfied iff

$$
\text { Ric }_{g}=c I+D \text {, }
$$

for some real number $c$ and $D \in \operatorname{Der}(\mathfrak{n})$, where $\operatorname{Der}(\mathfrak{n})$ denotes the Lie algebra of derivations of $\mathfrak{n}$. Note that this condition immediately implies the existence of a symmetric derivation. In particular, as pointed out in 19, we cannot solve the soliton equation over a characteristically nilpotent Lie group. Recall that a Lie algebra $\mathfrak{n}$ is characteristically nilpotent iff $\operatorname{Der}(\mathfrak{n})$ is nilpotent. Here we just notice that this obstruction does not apply to the class of 2-step nilpotent Lie algebras. In fact a characteristically nilpotent Lie algebra is at least 4-step nilpotent, for more details see 22]. Moreover there are no examples of characteristically nilpotent Lie algebras in dimension less or equal that six, as follows from [25].

It is now interesting to consider the equation in (3) on a non-nilpotent Lie group $G$, and using the 1-parameter group of diffeomorphisms generated by $D$ show that $g$ is a Ricci soliton. This fact is actually used by Lauret to exhibit some examples of non-nilpotent solvable Lie groups which admit a Ricci soliton structure. Solitons satisfying 3 are also called algebraic solitons, for more details see [21, [17] and the bibliography therein.

Finally, we point out that the soliton condition on a nilpotent Lie group can be neatly characterized in terms of metric solvable extensions.

Theorem 1.1 (Lauret). Let $N^{n}$ be a simply connected nilpotent Lie group with Lie algebra $\mathfrak{n}$ and left invariant metric $g$. Then $\left(N^{n}, g\right)$ admits a Ricci soliton structure iff $(\mathfrak{n}, g)$ admits a standard metric solvable extension $(\mathfrak{s}=\mathfrak{a} \oplus \mathfrak{n}, \tilde{g})$ whose corresponding simply connected Lie group $(S, \tilde{g})$ is Einstein.

Recall that a metric solvable extension of $(\mathfrak{n}, g)$ is a metric solvable Lie algebra $(\mathfrak{s}=\mathfrak{a} \oplus \mathfrak{n}, \tilde{g})$ such that $[\mathfrak{s}, \mathfrak{s}]_{\mathfrak{s}}=\mathfrak{n}=\mathfrak{a}^{\perp},\left.[,]_{\mathfrak{s}}\right|_{\mathfrak{n} \times \mathfrak{n}}=[,]_{\mathfrak{n}},\left.\tilde{g}\right|_{\mathfrak{n} \times \mathfrak{n}}=g$. The metric solvable extension is called standard if $\mathfrak{a}$ is abelian. Although Lauret results are non-costructive, the characterization given in Theorem 1.1 can be easily used to provide examples. For instance, any generalized Heisenberg Lie group (H-type) [3] and many 2-step nilpotent Lie groups admit a Ricci soliton structure, see [20] for a complete list of all the known examples.

With what regard to the uniqueness of nilpotent Ricci solitons, the following theorem provides a complete answer.

Theorem 1.2 (Lauret). Let $N^{n}$ be a simply connected nilpotent Lie group with Lie algebra $\mathfrak{n}$ and left invariant metric $g$. If $g$ and $g^{\prime}$ are left invariant Ricci soliton metrics, then there exist $c>0$ and $\eta \in A$ Aut $(\mathfrak{n})$ such that $g^{\prime}=c \eta(g)$.

Remark 1.3. Interestingly, in a recent preprint [17], Jablonski has extended many of the results of Lauret to the class of solvmanifolds. Remarkably, a Ricci solvsoliton has to be an algebraic Ricci soliton. Moreover, these solitons enjoy nice uniqueness properties as in the nilpotent case. For more details the reader is referred to [17] and to the bibliography therein.

The first explicit construction of Lauret solitons has been obtained by Baird and Danielo in [1]. In [1, the authors study 3-dimensional Ricci solitons which projects via a semi-conformal mapping to a surfaces and obtain a complete description of the soliton structures on $\mathrm{Nil}^{3}$ and $\mathrm{Sol}^{3}$ predicted by Lauret. 
In 23, Lott uses these particular soliton structures to study the long time behavior of type III Ricci flow solutions and also gives, among many other results, some explicit examples of four dimensional homogeneous soliton solutions, e.g. on $\mathrm{Nil}^{4}$. It turns out that all these solitons are expanding and of non-gradient type. These are the first known examples of non-gradient Ricci solitons. We also note that the property to be non-gradient plays a important role in the analytical study of the linear stability of these solutions, see [9].

In this paper, we concentrate on the study of the generic properties of homogeneous Ricci solitons providing a new point of view on the results found by Danielo, Baird and Lott.

\section{The scalar Curvature of a homogeneous Ricci soliton}

In this section we study the evolution of the scalar curvature on a homogeneous Ricci soliton. Recall that on a soliton solution to the Ricci flow the scalar curvature satisfies the equation

$$
R(t)=\frac{R_{0}}{\sigma(t)}=\frac{R_{0}}{1+2 \lambda t} .
$$

In particular if the initial scalar curvature $R_{0}$ is constant it stays constant during the evolution. In this case $R_{t}$ satisfies the simple ODE

$$
\frac{\partial R}{\partial t}=\frac{2(-\lambda)}{R_{0}} R^{2}, R(0)=R_{0}
$$

As proved in [11, the scalar curvature of a general Ricci flow solution evolves according the heat type equation

$$
\frac{\partial R}{\partial t}=\Delta R+2|R i c|^{2}
$$

we conclude that if the soliton has constant initial scalar curvature with $\lambda=\frac{-R_{0}}{n}$ then it must be trivial. In particular a nontrivial soliton structure cannot be associated to a divergence free vector field $X$, as can be proved by considering the trace of (2). In summary we have:

Lemma 2.1. A complete vector field $X$ associated to a nontrivial constant curvature Ricci soliton satisfies $\operatorname{div}(X)=k$, where $k$ is a nonzero constant.

We can now use Lemma 2.1 to obtain a complete description of the compact case.

Theorem 2.2. There are no non-trivial compact solitons with constant scalar curvature.

Proof. Stokes' theorem.

With regard to the compact homogeneous case it is interesting to notice that Theorem 2.2 can also be derived from the fact that there is a natural scaling invariant quantity that is increasing along the flow.

Lemma 2.3. Let $\left(M^{n}, g(t)\right)$ be a compact homogeneous solution to the Ricci Flow, then $R(t) V(t)^{\frac{2}{n}}$ is monotonically increasing unless the initial manifold is Einstein.

Proof. Because of the diffeomorphism invariance of the Ricci tensor and the short time existence and uniqueness for the Ricci flow on compact manifolds [10, the flow preserves the isometries of the initial Riemannian manifold. We conclude that a compact homogeneous Riemannian manifold remains homogeneous during the 
flow. Using the standard variation formulas for the volume and the scalar curvature functions [10] we have

$$
\begin{aligned}
\frac{d}{d t} R(t) V(t)^{\frac{2}{n}} & =R^{\prime}(t) V(t)^{\frac{2}{n}}+\frac{2}{n} V(t)^{\frac{2-n}{n}} V^{\prime}(t) R(t) \\
& =2|R i c|^{2} V(t)^{\frac{2}{n}}-\frac{2}{n} R^{2} V(t)^{\frac{2}{n}} \\
& =2\left|R i c-\frac{R}{n} g\right|^{2} V(t)^{\frac{2}{n}}
\end{aligned}
$$

Note that, because of the existence of the solitons discovered by Lauret, there is no analogue of Lemma 2.3 in the noncompact homogeneous case. Nevertheless, we can use the monotonicity property of the scalar curvature to derive the following partial generalization of Theorem 2.2 .

Theorem 2.4. There are no steady non-compact Ricci solitons with constant scalar curvature.

Proof. By the formula

$$
\frac{d R(t)}{d t}=2|R i c|^{2}
$$

we have that the scalar curvature is increasing unless the initial metric is Ricci flat.

It is interesting to note that Theorems 2.2 and 2.4 immediately extend to rule out the existence of compact homogeneous and non-compact homogeneous steady breathers. For the definition of breather see [5]. This observation implies the following remark.

Remark 2.5. As pointed out in [10], the Ricci flow equation on a given homogeneous spaces reduces to an ODE on the finite dimensional moduli space of homogeneous metrics. Now the nonexistence of steady breathers then implies that these interesting geometrical ODEs have no periodic solutions, at least for those initial data that admit geometrical interpretation. For a detailed study of the Ricci flow ODEs on three and four dimensional homogeneous spaces we refer to [14] and [15].

Finally, we notice that the sign of the scalar curvature determines if the soliton is expanding or shrinking.

Lemma 2.6. Any constant scalar curvature soliton of expanding or shrinking type must have respectively negative or positive initial scalar curvature.

For more results concerning homogeneous shrinking solitons with positive scalar curvature the reader is referred to Remark 3.2 below.

\section{LEFT INVARIANT RiCCI SOLITONS}

In this section we concentrate on the study of the geometrical properties of left invariant Ricci solitons. First, we explain why all the known explicit examples are of expanding type, then we study the possibility to solve the soliton equation within the set of left invariant vector fields and then we show that in "general" a left invariant soliton cannot be of gradient type. We point out that we usually work on simply connected Lie group since a non-trivial soliton structure on a Lie group with non-trivial fundamental group can clearly be transported to its universal cover. 
3.1. The sign of the soliton constant. As noticed in Lemma 2.6 the sign of the scalar curvature determines the type of the soliton. The sign of the scalar curvature associated to a left invariant metric has been extensively studied by many authors, see in particular 24] and 2. We can then state the following.

Proposition 3.1. A left invariant soliton or breather structure on a solvable Lie group is necessarily expanding.

Proof. As proved in [24, any left invariant metric $g$ over a solvable Lie group $G$ is either flat or has strictly negative scalar curvature. If the scalar curvature negative, by Lemma 2.6 we have that the left invariant soliton structure (if any) must be of expanding type.

This simple result explains why the examples explicitly constructed by Baird, Danielo and Lott are of expanding type. Moreover it ensures that every nilsoliton predicted by Lauret must be of expanding type. The above lemma can actually be improved. In 24, Milnor studies the problem of which Lie groups endowed with left invariant metric admit positive scalar curvature. Using the Iwasawa decomposition theorem Wallach was able to give a sufficient condition, namely that the universal covering of the Lie group is not homeomorphic to an Euclidean space, for the details see 24. In 24 it is actually conjectured that this condition is also necessary. This conjecture turned to be true as shown by Bérard-Bergery in [2] We then have that a Lie group whose universal cover is homeomorphic to a Euclidean space can admit only expanding left invariant soliton structures. We also have that a non-compact shrinking left invariant soliton must be homeomorphic to a product of a compact Lie group with some Euclidean space.

Remark 3.2. It follows from results of Naber [26] and Petersen-Wylie [29] that any shrinking homogeneous Ricci soliton must be trivial. More precisely, it must be isometric to a product of a compact positive Einstein manifold with some Euclidean space. In fact, by Theorem 1.2. in [26] a shrinking homogeneous soliton must be of gradient type. Finally, by Theorem 1.1. in [29] such a soliton must be trivial. For more details see also Remark 3.8.

3.2. Nonsolvability for left invariant vector fields. We derive some generalities about the Ricci soliton equation over a metric $g$ that is left invariant. Recall that a metric $g$ is called left invariant if $L_{h}^{*} g=g$ for all $h \in G$ where $L_{h}$ is the left translation by $h$. Recall also that a vector field $X$ is called left invariant iff $L_{h}^{*} X=X$ for all $h \in G$. Consider now the Ricci soliton equation (2) and take the pull back by a left translation, we then get

$$
\begin{aligned}
& -2 L_{h}^{*} R i c_{g}=L_{h}^{*}\left(L_{X} g\right)+2 \lambda L_{h}^{*} g \\
& -2 R i c_{L_{h}^{*} g}=L_{L_{h}^{*} X}\left(L_{h}^{*} g\right)+2 \lambda g \\
& -2 R i c_{g}=L_{L_{h}^{*} X} g+2 \lambda g,
\end{aligned}
$$

which implies

$$
L_{\left(L_{h}^{*} X-X\right)} g=0 \text {. }
$$

We conclude that $L_{h}^{*} X-X$ is a Killing vector field for all $h \in G$, we then say that $X$ is left invariant modulo Killing fields or simply a left-Killing field. Following this terminology a trivial left-Killing field is just an ordinary left invariant vector field.

At this stage of the theory we cannot be sure of the existence of nontrivial leftKilling fields, indeed one may think to consider the usual left invariant vector fields in order to reduce the soliton condition to an algebraic set of equations on the Lie algebra of the group. It turns out that this is not possible in general.

Lemma 3.3. Any left invariant vector field over an unimodular Lie group is divergence free. 
Proof. First, we notice that any left invariant vector field has constant divergence. Let $X$ be a left invariant vector field over a Lie group $G^{n}$ equipped with a left invariant metric $g$ and let $\left\{e_{i}\right\}_{i=1}^{n}$ be a left invariant global orthonormal frame. We can then compute the divergence as follows

$$
\operatorname{div}(X)=\sum_{i} g\left(\nabla_{e_{i}} X, e_{i}\right)
$$

and notice that is constant since the $\left\{\nabla_{e_{i}} X\right\}$ are left invariant. Recall now that a Lie group is called unimodular if its left invariant Haar measure $\omega$ is also right invariant. Recall also that given a left invariant vector field $X$ its flow can be explicitly written in terms of right translations and the exponential map, namely for any $x \in G^{n}$ we have

$$
F_{t}(x)=R_{\exp (\mathrm{tX})}(x) .
$$

Let $d \omega$ the left invariant volume form associated to the Haar measure. Since we are assuming $d \omega$ to be also right invariant we clearly have $L_{X} d \omega=0$. We conclude recalling the identity $L_{X} d \omega=\operatorname{div}(X) d \omega$.

We can now combine the above result with Lemma 2.1 to obtain the following proposition.

Proposition 3.4. For any unimodular Lie group the homogeneous soliton equation cannot be solved within the set of left invariant vector fields.

Note that Proposition 3.4 implies the existence of nontrivial left-Killing fields. Recall that, in terms of the Lie algebra $\mathfrak{g}$, the unimodular condition is equivalent to requiring that the linear transformation ad $x$ has trace zero for every $x \in \mathfrak{g}$. We conclude that any nilpotent Lie group is unimodular which implies, by Proposition 3.4 the existence of at least one complete nontrivial left-Killing field on any nilsoliton.

We now briefly study the same question on non-unimodular Lie groups. Notice that on a non-unimodular Lie group the divergence of a left invariant vector field can be different from zero. Let $(\mathfrak{g},\langle\rangle$,$) be a three dimensional non-unimodular metric$ Lie algebra. As shown in Section 6 of [24], we can always find an orthonormal basis $e_{1}, e_{2}, e_{3}$ so that

$$
\begin{aligned}
& {\left[e_{1}, e_{2}\right]=\alpha e_{2}+\beta e_{3}} \\
& {\left[e_{1}, e_{3}\right]=\gamma e_{2}+\delta e_{3}}
\end{aligned}
$$

with $\left[e_{2}, e_{3}\right]=0, \alpha+\delta \neq 0$ and $\alpha \gamma+\beta \delta=0$. Following the literature we refer to this special frame as a Milnor frame (M-frame). The divergence of the elements in the M-frame is easily computed:

$$
\begin{aligned}
\operatorname{div}\left(e_{1}\right) & =g\left(\nabla_{e_{1}} e_{1}, e_{1}\right)+g\left(\nabla_{e_{2}} e_{1}, e_{2}\right)+g\left(\nabla_{e_{3}} e_{1}, e_{3}\right) \\
& =0+\frac{1}{2}\left\{g\left(\left[e_{2}, e_{1}\right], e_{2}\right)-g\left(\left[e_{1}, e_{2}\right], e_{2}\right)+g\left(\left[e_{2}, e_{2}\right], e_{1}\right)\right\} \\
& +\frac{1}{2}\left\{g\left(\left[e_{3}, e_{1}\right], e_{3}\right)-g\left(\left[e_{1}, e_{3}\right], e_{3}\right)+g\left(\left[e_{3}, e_{3}\right], e_{1}\right)\right\} \\
& =-(\alpha+\delta) ;
\end{aligned}
$$

an analogous compututation shows that $\operatorname{div}\left(e_{2}\right)=\operatorname{div}\left(e_{3}\right)=0$. In summary for any orthonormal M-frame $e_{1}$ has nonzero divergence while $e_{2}$ and $e_{3}$ are divergence free. Assume now the soliton equation can be solved within the set of left invariant vector fields. Let $(\mathfrak{g},\langle\rangle$,$) our soliton metric Lie algebra, and let e_{1}, e_{2}, e_{3}$ the associated M-frame. Notice that the left invariant vector field that solve the soliton condition must have a nonzero component in the direction of $e_{1}$. Using Lemma 6.5. 
in 24, it is easy to express the Ricci tensor in terms of the constants of structure of the Lie algebra. Using this fact and performing analogous computations for the Lie derivative of the metric we reduce the soliton condition $-2 R i c_{g}=2 \lambda g+L_{X} g$, where $X=a e_{1}+b e_{2}+c e_{3}$ with $a \neq 0$, to the following set of algebraic equations

$$
\begin{aligned}
& -2\left(\begin{array}{ccc}
-\alpha^{2}-\delta^{2}-\frac{1}{2}(\beta+\gamma)^{2} & 0 & 0 \\
0 & -\alpha(\alpha+\delta)+\frac{1}{2}\left(\gamma^{2}-\beta^{2}\right) & 0 \\
0 & 0 & -\delta(\alpha+\delta)+\frac{1}{2}\left(\beta^{2}-\gamma^{2}\right)
\end{array}\right) \\
& =2 \lambda\left(\begin{array}{lll}
1 & 0 & 0 \\
0 & 1 & 0 \\
0 & 0 & 1
\end{array}\right)+a\left(\begin{array}{ccc}
0 & 0 & 0 \\
0 & -2 \alpha & -(\gamma+\beta) \\
0 & -(\gamma+\beta) & -2 \delta
\end{array}\right) \\
& +\left(\begin{array}{ccc}
0 & b \alpha+c \delta & b \beta+c \delta \\
b \alpha+c \delta & 0 & 0 \\
b \beta+c \delta & 0 & 0
\end{array}\right)
\end{aligned}
$$

This system is easily reduced to

$$
\begin{aligned}
& 2\left(\alpha^{2}+\delta^{2}\right)=2 \lambda \\
& 2 \alpha(\alpha+\delta)=2 \lambda-2 a \alpha \\
& 2 \delta(\alpha+\delta)=2 \lambda-2 a \delta .
\end{aligned}
$$

Thus, $\lambda=\alpha^{2}+\delta^{2}$ which implies, under the assumption $\delta \neq \alpha, a=-(\alpha+\delta)$. Substituting this value in the second of the equations above we obtain $\delta=\alpha=0$ that is a contradiction. In the remaining case $\delta=\alpha$ an easy computation shows that $a=0$ which clearly implies the triviality of the soliton. In summary we proved the following proposition.

Proposition 3.5. For any three dimensional non-unimodular Lie algebra the soliton equation cannot be solved within the set of left invariant vector fields.

We don't know if a similar restriction holds in dimension greater than three.

3.3. Left invariant gradient solitons. As mentioned in the introduction, all the known explicit constructions of left invariant Ricci solitons are of non-gradient type. Remarkably these are the first examples of non-gradient Ricci solitons. We now show that this property is indeed generic for this class of solitons.

Recall that the gradient Ricci soliton equation is given by

$$
R i c_{g}+\nabla \nabla f+\frac{\lambda}{2} g=0
$$

We then say that $g$ is an expanding, shrinking, or steady soliton if $\lambda>0, \lambda<0$ or $\lambda=0$ respectively. By standard manipulations in (8) we can derive the following useful identities

$$
\begin{gathered}
R+\Delta f+\frac{\lambda}{2} n=0 \\
d R=2 \operatorname{Ric}(\nabla f, \cdot) \\
\Delta R=g(\nabla R, \nabla f)-\lambda R-2|R i c|^{2},
\end{gathered}
$$

for a proof see [5]. Using identity (9), we notice that if $R$ is constant then $\Delta f$ is constant. We can then derive another proof of theorem 2.2. In fact, by Perelman's result on compact Ricci solitons we can restrict our attention to shrinking gradient solitons. Thus, using the nonexistence of sub/superharmonic functions on compact 
manifolds we can rule out again the constant scalar curvature case. Combining now identities (9) and (11), we have

$$
\frac{2}{n}(R+\Delta f) R=2|R i c|^{2}
$$

that implies

$$
\frac{2}{n} \Delta f R=2\left|R i c-\frac{R}{n} g\right|^{2} .
$$

We conclude that on a gradient shrinking soliton with constant scalar curvature $\Delta f$ and $R$ have the same sign. Finally, using 10 we can derive a nice rigidity property for constant scalar curvature solitons. Indeed, using the identity

$$
0=d R=2 \operatorname{Ric}(\nabla f, \cdot),
$$

we have that if the Ricci curvature has definite sign then the soliton must necessarily be Einstein. More precisely we can state the following:

Proposition 3.6. A Riemannian manifold with constant scalar curvature and non degenerate Ricci tensor cannot be a gradient Ricci soliton.

We conclude our study showing that Proposition 3.6 can actually be improved if we restrict to left invariant solitons. Formula (13) combined with the Bochner identity for $|\nabla f|^{2}$ gives

$$
\begin{aligned}
\Delta|\nabla f|^{2} & =2|\nabla \nabla f|^{2}+2 \operatorname{Ric}(\nabla f, \nabla f)+2 g(\nabla f, \nabla \Delta f) \\
& =2|\nabla \nabla f|^{2},
\end{aligned}
$$

then if we further assume $|\nabla f|^{2}=c$, with $c$ a constant, we derive that the soliton is trivial. Thus, since

$$
L_{h}^{*} \nabla_{g} f=\nabla_{L_{h}^{*} g}\left(f \circ L_{h}\right)=\nabla_{g}\left(f \circ L_{h}\right),
$$

we have that for at least some $h \in G$ the vector field $\nabla_{g}\left(f \circ L_{h}-f\right)$ is a nontrivial Killing field. Now, a gradient Killing field must be parallel. We conclude that our group splits locally as a Riemannian product with an interval. The simple observation that a gradient soliton structure on Lie group lifts to its universal Riemannian cover immediately implies the following theorem.

Theorem 3.7. Any metric Lie algebra $(\mathfrak{g},\langle\rangle$,$) with trivial Euclidean de Rham$ factor cannot be a gradient Ricci soliton.

Remark 3.8. It follows from a rigidity result of Petersen-Wylie [29] that any homogeneous gradient Ricci soliton must have a non-trivial Euclidean deRham factor. The reader should compare Theorem 3.7] with the more general Theorem 1.1. in [29]. The first version of this work and the preprint of [29] appeared independently on the arXiv in October $200 \%$.

We can now apply Proposition 3.6 to the class of nonsingular 2-step nilpotent Lie algebras.

Theorem 3.9. A nonsingular 2-step nilpotent Lie algebra cannot admit a left invariant gradient soliton structure.

Proof. Recall that a 2 -step nilpotent Lie algebra $\mathfrak{n}$ is called nonsingular if the map ad $x: \mathfrak{n} \rightarrow \mathfrak{z}$ is surjective for all $x \in \mathfrak{n}-\mathfrak{z}$, where $\mathfrak{z}$ is the center of $\mathfrak{n}$. Now, on a general 2-step nilpotent Lie algebra equipped with a positive definite inner product $\langle$,$\rangle , denoted by \mathfrak{v}$ the orthogonal complement of $\mathfrak{z}$ in $\mathfrak{n}$, we can define for each $z \in \mathfrak{z}$ a skew symmetric linear transformation $j(z): \mathfrak{v} \rightarrow \mathfrak{v}$ by

$$
j(z) x=(\operatorname{ad} x)^{*} z \text { for all } x \in \mathfrak{v}
$$


where $(a d x)^{*}$ denotes the adjoint of $a d x$. As extensively shown by many authors, see in particular [7] and [3, most of the geometry of the metric Lie algebra $\{\mathfrak{n},\langle\rangle$, is given by the properties of the maps $j(z)$. In particular the kernel of the Ricci tensor, seen as a symmetric linear transformation on the Lie algebra, is given by the following subspace of the center $\{z \in \mathfrak{v} \mid j(z)=0\}$, see Proposition 2.5 in [7. Being $\mathfrak{n}$ nonsingular, an easy argument shows that $j(z): \mathfrak{v} \rightarrow \mathfrak{v}$ is nonsingular for any nonzero $z \in \mathfrak{z}$. We conclude that for any nonsingular 2 -step nilpotent metric Lie algebra $\{\mathfrak{n},\langle\rangle$,$\} the associated Ricci tensor is non-degenerate. The claim is now$ a consequence of Theorem 3.7 .

We then have the following theorem for generalized Heisenberg Lie groups $(\mathrm{H}-$ type).

Theorem 3.10. Any simply connected metric H-type Lie group admits an expanding non-gradient left invariant Ricci soliton structure.

Proof. Recall that a 2-step nilpotent metric lie algebra $\{\mathfrak{n},\langle\rangle$,$\} is of Heisenberg$ generalized type if $j(z)^{2}=-|z|^{2} i d_{\mathfrak{v}}$ for all $z \in \mathfrak{z}$. This clearly implies that for any $z \in \mathfrak{z}$ the linear map $j(z)$ is nonsingular. Now a routine linear algebra argument shows that the Lie algebra $\mathfrak{n}$ must be nonsingular. By Theorem 3.9 we conclude that a H-type Lie group cannot be a gradient Ricci soliton. For what regard the existence we follow Lauret. The standard metric solvable extension of the H-type Lie groups are exactly the harmonic Damek-Ricci spaces that are solvable and Einstein, see for example [3. By Theorem 1.1 we conclude that these groups are algebraic Ricci solitons. Finally, using Proposition 3.1 we have that these structures must be of expanding type.

\section{FinAl REMARKS}

Theorem 3.10 provides the first example of a countably infinite family of nongradient Ricci solitons. Recall that for any $n \in \mathbb{N}$ there exist a countably infinite number of non-isomorphic $\mathrm{H}$-type Lie algebras with $\operatorname{dim} \mathfrak{z}=n$, see again [3]. It is also interesting to notice that any H-type group admits a lattice, i.e., a cocompact discrete subroup. This result is easily derived from the existence of an integral structure on any H-type Lie algebra, see [6]. In fact a well known result by Malcev, see for example 30, ensures that a simply connected nilpotent Lie group admits a lattice iff its Lie algebra admits a rational structure. Thus, let $N$ be a H-type Lie group and $\Gamma$ a lattice. We then have that the left-Killing vector field $X$ is not preserved by the action of the subroup $\Gamma$ otherwise we could project the soliton structure on $(N, g)$ to a well defined homogeneous soliton structure on the compact manifold $(N / \Gamma, \tilde{g})$. Thus, Theorem 3.10 also provides an infinite family of pseudosolitons, i.e., compact manifold with no soliton structure that acquire one when lifted to the universal cover, see [9] for the definition of pseudosolitons.

As a final application we derive that the 3-dimensional non-product geometries $\mathrm{Nil}^{3}, \mathrm{Sol}^{3}$ and $\mathrm{SL}(2, \mathbb{R})$ do not admit any left invariant gradient soliton structure, since any left invariant metric on these groups is de Rham indecomposable, see [31. Note how a similar result cannot be obtained by using Proposition 3.6. In fact, as proved in 24 , the signature of the Ricci form on both $\operatorname{Sol}^{3}$ and $\operatorname{SL}(2, \mathbb{R})$ can be either $(+,-,-)$ or $(0,0,-)$. More in general Theorem 3.7 provides a satisfactory obstruction in the case of irreducible left invariant solitons.

It is interesting to note that very recently Jablonski in [17] has finally rule out the existence of a homogeneous Ricci soliton structure on $\operatorname{SL}(2, \mathbb{R})$. This result, in a sense, closes up the connection between the theory of homogeneous Ricci solitons and the special geometries of Thurston. 
Finally, I would like to point out that since the appearance of first version of this work on the arXiv, the theory of homogeneous Ricci solitons has vigorously grown. The interested reader should for example consult [8], [16], 27], 20, [15], 17], 29], 26, 21 .

Acknowledgements. I would like to thank Jorge Lauret for his kind interest in this work and for several constructive comments. Moreover, I would like to thank Professor John Milnor for several useful discussions regarding 24. I also would like to thank the referee for guiding me through the most recent literature on homogeneous solutions of the Ricci flow and for pertinent comments on this manuscript.

\section{REFERENCES}

[1] P. Baird, L. Danielo Three-dimensional Ricci solitons which project to surfaces. J. Reine Angew. Math. 2007, 65-71.

[2] L. Bérard-Bergery, Sur la curbure de métriques riemanniennes invariantes des groupes de Lie et des espaces homogènes. Ann. Sci. École Norm. Sup. 11 (1978), no. 4, 543-576.

[3] J. Berndt, F. Tricerri, L. Vanhecke, Generalized Heisenberg groups and Damek-Ricci harmonis spaces. Lect. Notes in Math. 1598, Springer-Verlag Berlin Heidelberg, 1995.

[4] R. Bryant, Unpublished result on Ricci solitons.

[5] B. Chow et al., The Ricci Flow: Techniques and Applications. Part I. Mathematical Surveys and Monographs, AMS, Providence, RI, 2007.

[6] G. Crandall, J. Dodziuk, Integral Structures on H-type Lie Algebras. J. Lie Theory 12 (2002), no.1, 69-79.

[7] P. Eberlein, Geometry of 2-step nilpotent groups with a left invariant metric. Ann. Sci. École Norm. Sup. 27 (1994), no. 5, 611-660.

[8] D. Glickenstein, T. L. Payne, Ricci flow on three-dimensional, unimodular metric Lie algebras. Comm. Anal. Geom. 18 (2010), no. 5, 927-961.

[9] C. Guenther, J. Isenberg, D. Knopf, Linear stability of homogeneous Ricci solitons. Int. Math. Res. Not. (2006), Art. ID96253, 1-30.

[10] R. S. Hamilton, Three-manifolds with positive Ricci curvature. J. Differential Geometry 17 (1982), no. 2, 255-306.

[11] R. S. Hamilton, The Ricci flow on surfaces. Mathematics and general relativity (Santa Cruz, CA, 1986), 237-262, Contemp. Math., 71, Amer. Math. Soc., Providence, RI, 1988.

[12] T. Ivey, Ricci solitons on a compact three-manifold. Diff. Geom. Appl. 3 (1993), 301-307.

[13] T. Ivey, New examples of complete Ricci solitons. Proc. Amer. Math. Soc. 122 (1994), 241245.

[14] J. Isenberg, M. Jackson, Ricci flow on locally homogeneous geometries on closed manifold. J. Differential Geometry 35 (1992), no. 3, 723-741 .

[15] J. Isenberg, M. Jackson, P. Lu, Ricci flow on locally homogeneous 4-manifolds. Comm. Anal. Geom. 14 (2006), 345-386.

[16] M. Jablonski, Concerning the existence of Einstein and Ricci soliton metrics on solvable Lie groups. Geometry \& Topology 15 (2011), no. 2, 735-764.

[17] M. Jablonski, Homogeneous Ricci solitons. arXiv:1109.6556, (2011).

[18] N. Koiso, On rotationally symmetric Hamilton's equation for Kähler-Einstein metrics. Recent Topics in differential and analytic geometry, 327-337, Adv. Studies Pure Math., 18-I, Academic Press, Boston, MA, 1990.

[19] J. Lauret, Ricci solitons homogeneous nilmanifolds. Math. Ann. 319 (2001), no. 4, 715-733.

[20] J. Lauret, Minimal metrics on nilmanifolds. Differential geometry and its applications, 79-97, Mathyzpress, Prague, (2005).

[21] J. Lauret, Ricci soliton solvmanifolds. J. Reine Angew. Math. 650 (2011), 1-21.

$[22]$ G. F. Leger, S. Togo, Characteristically nilpotent Lie algebras. Duke Math. J. 26 (1959), 623-628.

[23] J. Lott, On the long time-time behavior of type-III Ricci flow solutions. Math. Ann. 339 (2007), 627-666.

[24] J. Milnor, Curvatures of left invariant metrics on Lie groups. Advances in Math. 21 (1976), no. 3, 293-329.

[25] V. V. Morozov, Classification of nilpotent Lie algebras of sixth order (in Russian). Izv. Vyssh. Ucheb. Zar. 4 (1958), no. 4, 161-171. 
[26] A. Naber, Noncompact shrinking four solitons with nonnegative curvature. arXiv:0710.5579, (2008).

[27] T. Payne, The Ricci flow for nilmanifolds. J. Mod. Dyn 4 (2010), no. 1, 65-90.

[28] G. Perelman, The entropy formula for the Ricci flow and its geometric applications. arXiv:0211.1159, (2002).

[29] P. Petersen, W. Wylie, On gradient Ricci solitons with symmetry. Proc. Amer. Math. Soc. 137 (2009), no. 6, 2085-2092.

[30] M. S. Raghunathan, Discrete Subgroups of Lie Groups. Springer, New York 1972.

[31] P. Scott, The geometries of 3-manifold. Bull. London Math. Soc. 15 (1983), 401-487.

Department of Mathematics, Duke University, Box 90320, Durham, NC 27708-0320, USA

E-mail address: luca@math.duke.edu 